\title{
Numerical study of mixing index in offset inlets 3-D T mixer with bend shape mixing channel
}

\author{
Ranjan Prakash*, Mohammad Zunaid and Samsher \\ Department of Mechanical Engineering, Delhi Technological University, Delhi, India \\ * Corresponding Author : ranjanprakashmnnit@gmail.com
}

\begin{abstract}
The objective of this paper is the computational analysis on the mixing index of simple $\mathrm{T}$ shape mixer, offset inlets $\mathrm{T}$ shape mixer, and offset inlets $\mathrm{T}$ mixer with bend shape mixing channel by CFD simulation. Computational fluid dynamics software package solves conservation of mass equation, conservation of momentum equation, and conservation of energy equation. In the case of offset inlets T shape mixer, as the aspect ratio (height to width ratio) of mixing channel increased so mixing quality also increased and offset inlets $\mathrm{T}$ mixer with bend shape is a combination of increased aspect ratio as well as chaotic advection mechanisms, so it provides advanced mixing index than offset inlets T shape mixer and simple T shape mixer. Pressure fall in offset inlets T shape mixer is excess than simple T shape mixer but narrowly degraded than offset inlets T mixer with bend shape. Chaotic advection rooted microchannel generates secondary flow because of which motives a high-pressure drop in the microchannel.
\end{abstract}

Keywords: Chaotic advection; Fluent; Mixing quality; Passive micromixer; Simulation.

\section{INTRODUCTION}

Microfluidics research develops two inventions viz Micro-Total Analysis System and Lab on a Single Chip, which is plied in the biomedical and chemical industries for sample preparation and analysis, DNA sequencing, synthesis of nucleic acids, enzyme reactions, drug delivery and sequencing, protein folding, cell separation and detection, etc. Micromixers can be separated into two different types namely: active mixer and passive mixer. Active mixers use external powers like Magnetic force, Electric field force, Thermal energy, Pressure gradient, Acoustic wave, etc. Another kind of micromixer is a passive mixer. Passive mixers depend on the special designs of the microchannel to mix the fluids without using any external powers. From the literature, in the case of passive mixers, the highest mixing index is obtained by dividing and recombination process and generating chaotic advection mechanism. SAR process means fluids to be fritted is divide and recombined to renovate the diffusion process. Microchannel based on the SAR process is more effective at low Re. The concept of Chaotic advection is created by either kilt/slant the mixing channel or insert obstacles in the mixing channel. Microchannel based on the chaotic advection mechanism is more effective at large Re.

In this concern, Quiroz et al. (2016) examined the impact of aspect ratio (height to width ratio) of mixing channel of offset inlets T shape micromixer in Reynolds number extent from 50 to 700. Quiroz et al. (2014) analyzed the three-dimensional $\mathrm{T}$ shape mixer and likened the mixing index value with the typical $\mathrm{T}$ shape mixer. Solehati et al. (2014) calculated the mixing index of a microchannel $\mathrm{T}$ junction with a wavy curvature and introduced the performance index for microchannel. Rahimi et al. (2014) studied the impact of different confluence angles and discharge rate ratios of five asymmetrical micromixers. Nguyen et al. (2008) proposed a Y-shape 
mixer with the square-wave channel having a square obstacle. Tsai et al. (2009) proposed a Y-junction microchannel to study the mixing phenomena between water and Ferro-nanofluid. Wong et al. (2004) fabricated a $\mathrm{T}$ shape microchannel to investigate their fast mixing feasibility by experimental analysis and numerical simulations. Alam et al. (2012) introduced a special design micromixer(with circular mixing zone) and compared the mixing quality of this micromixer with the other three planar micromixers. Hossain et al. (2009) investigated mixing quality of three inactive microchannels. X. Chen et al. (2016) estimated the mixing index of three reptile micromixers by CFD simulation and experimental analysis. Hsieh et al. (2013) examined the mixing performance of Y-junction microchannels located at different angles by optical technique. Tokas et al. (2020) examined the mixing capacity of the T shape mixer with helical structure and T- junction straight. Tokas et al. (2020) examined mixing phenomena in T-junction microchannel with Newtonian and Non-newtonian fluids. Javad et al. (2020) screened mixing phenomena of aluminum oxide with water in a spiral T shape mixer. Wang et al. (2013) proposed a microchannel in the form of a tree shape for rapid mixing. Vatankhah et al. (2018) analyzed the parametric study of the plane spiral shape mixer. Dauyeshova et al. (2018) analyzed the effect of cylindrical and rectangular obstacles in the T-junction micromixer. Veldurthi et al. (2015) demonstrated the mixing phenomena in the cylindrical chamber having a micro-rotor at the top of the chamber by numerical simulation. Lobasov et al. (2018) evaluated mixing quality in a T shape mixer for various fluids characteristics by CFD. Liu et al. (2019) studied concerning nanoparticles mixed with water in a $\mathrm{T}$ shape mixer. Chen et al. (2016) proposed two micro-channels SESM and FESM to achieve a high value mixing index. Haghighinia et al. (2019) analyzed a T junction microchannel having a rectangular winglet in mixing channel. Rahmannezhad et al. (2019) renovated Y-shape grooved micro-channel with a circular, diamond shape, and square shape obstructions. Chen et al. (2017) designed a multi-units obstacle microchannel and likened the mixing index value with the T shape mixer. Nazari et al. (2019) designed a microchannel keeping spherical, square, trigonic, and rhombus mixing chambers with conductive surfaces. Silva et al. (2017) introduced a T shape mixer with different inlets width and read outed the impact of pulsed signals enforced at inlets. Santana et al. (2017) designed microchannel reactors with static mixing elements for biodiesel synthesis. Dundi et al. (2019) studied about swirl effect in a passive T-micromixer. Boye et al. (2020) designed and modeled transient flows in a crude oil pipeline system. Sahnoune et. al. (2020) simulated Newtonian Drilling Mud flow in the turbine.

Afzal et al. (2019) proposed a caloric investigation of water and coolant in a spiral reel heat exchanger.

Considering the literature survey, in this paper simple $\mathrm{T}$ shape mixer, offset inlets $\mathrm{T}$ shape mixer, and offset inlets $\mathrm{T}$ mixer with bend shape is proposed and likened the mixing index of simple T shape mixer, offset inlets $\mathrm{T}$ shape mixer, and offset inlets T mixer with bend shape.

\begin{tabular}{|ll|}
\hline Nomenclature & \\
$\mathbf{u}=$ velocity vector & $\boldsymbol{v}=$ kinematic viscosity \\
$\operatorname{Re}=$ Reynolds number & $\boldsymbol{\sigma}=$ variance \\
$\boldsymbol{\mu}=$ dynamic viscosity of fluid & $\mathrm{P}=$ pressure \\
$\mathrm{D}=$ diffusion coefficient & $\mathrm{C}=$ concentration \\
\hline
\end{tabular}




\section{MICROMIXER STRUCTURE}

Simple T shape mixer, offset inlets T shape mixer, and offset inlets T mixer with bend shape are shown in figure 1. Water and water-soluble dye enter into the channel from the right and left inlets respectively. For comparison purpose, the hydraulic diameter of inlets channel and mixing channel of all three geometry were taken same.

Table 1. Geometry details of Simple T-shape mixer, offset inlets T-shape mixer and offset inlets

T-micromixer with bend shape.

\begin{tabular}{|l|c|c|c|}
\hline \multicolumn{1}{|c|}{ Type } & $\begin{array}{c}\text { Simple T-junction } \\
\text { (All dimension in } \boldsymbol{\mu m} \text { ) }\end{array}$ & $\begin{array}{c}\text { offset inlets T-junction } \\
\text { (All dimension in } \boldsymbol{\mu m} \text { ) }\end{array}$ & $\begin{array}{c}\text { offset inlets T-junction } \\
\text { with bend structure } \\
\text { (All dimension in } \boldsymbol{\mu m} \text { ) }\end{array}$ \\
\hline $\begin{array}{l}\text { Total length of inlets } \\
\text { channel }\end{array}$ & 800 & 800 & 800 \\
\hline Mixing channel length & 3000 & $\sim 3000$ & $\sim 3000$ \\
\hline $\begin{array}{l}\text { Inlets channel cross- } \\
\text { sectional area }\end{array}$ & $100 * 100$ & $150 * 75$ & $150 * 75$ \\
\hline $\begin{array}{l}\text { Mixing channel cross- } \\
\text { sectional area }\end{array}$ & $100 * 200$ & $200 * 100$ & $100 * 100$ \\
\hline $\begin{array}{l}\text { Hydraulic diameter of } \\
\text { inlets channel }\end{array}$ & 100 & 100 & $400 / 3$ \\
\hline $\begin{array}{l}\text { Hydraulic diameter of } \\
\text { mixing channel }\end{array}$ & $400 / 3$ & $400 / 3$ & 100 \\
\hline
\end{tabular}

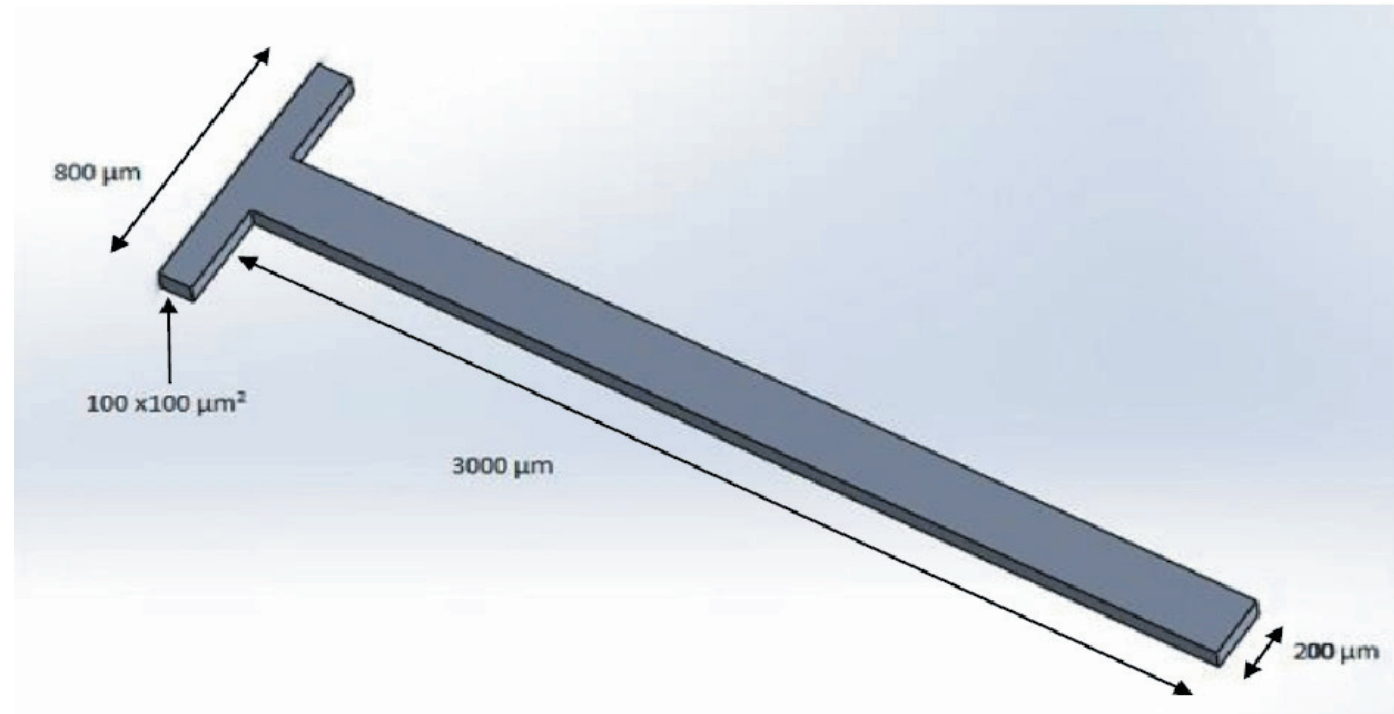

(a) 


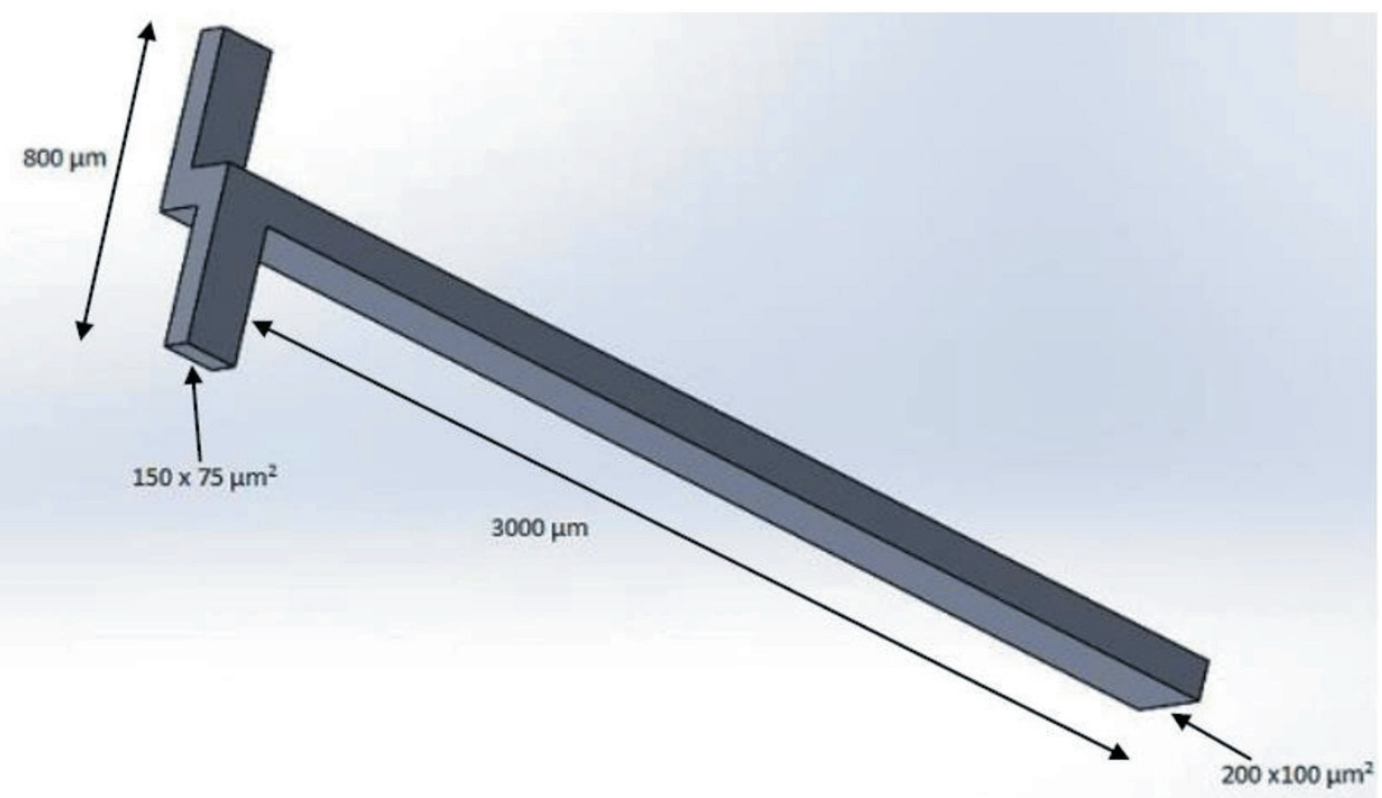

(b)

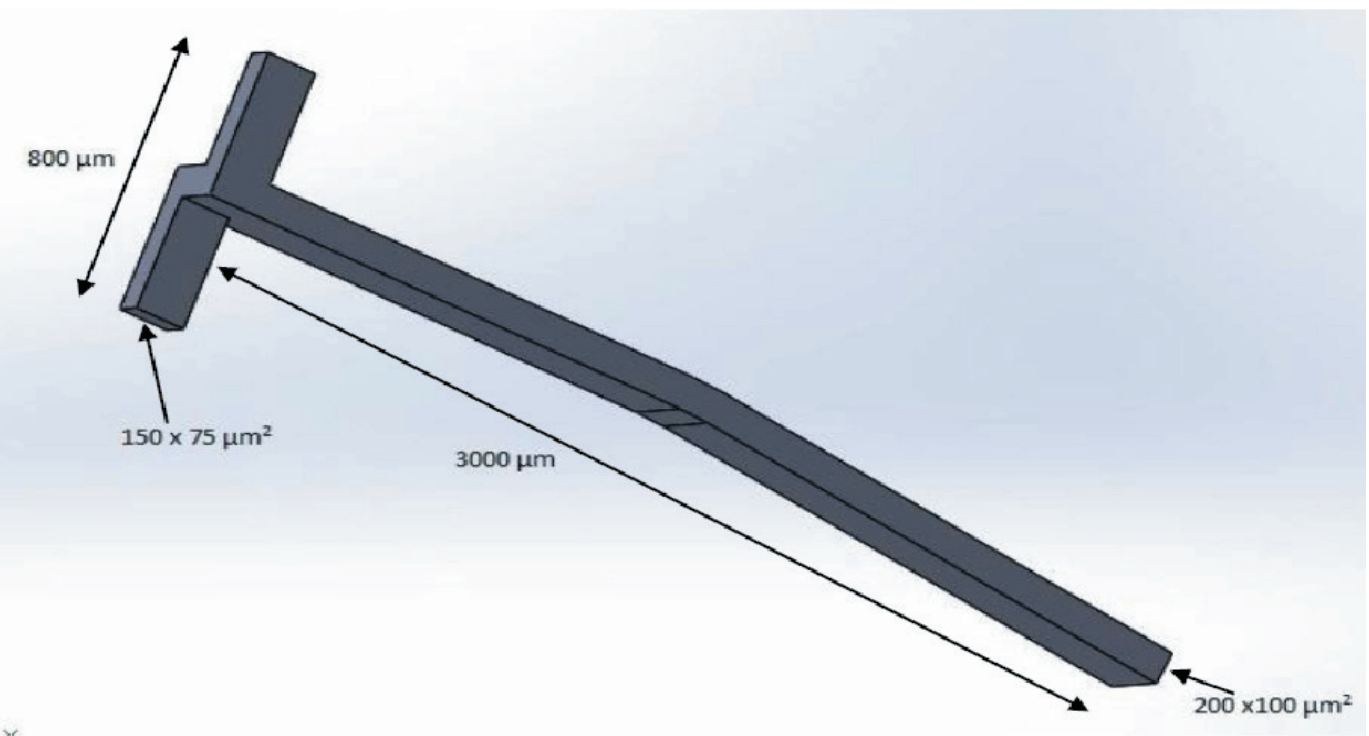

(c)

Figure1. Geometry of micro-channel T-junction design:

(a) simple $T$ shape mixer

(b) offset inlets $\mathrm{T}$ shape mixer

(c) offset inlets T mixer with bend structure. 


\section{SIMULATION DETAILS}

The flow into the microchannels was simulated by CFD software, ANSYS. This software is rely on the FVM. In ANSYS software, Fluent solves the momentum equation, energy equation, and conservation of mass equation. The equations of conservation are characterized in Balasubramaniam et al. (2017) and demonstrated as from equation (1) to equation (3):

$$
\begin{gathered}
\text { Continuity equation: } \nabla . \mathrm{u}=0 \\
\text { Navier-Stokes equation: } \frac{\partial u}{\partial t}+\mathrm{u} \cdot \nabla u=-\frac{1}{\rho} \nabla p+\mathrm{v} \nabla^{2} \mathrm{u} \\
\text { Convection-Diffusion equation: } \frac{\partial c}{\partial t}+(\mathrm{u} . \nabla) \mathrm{c}=\mathrm{D} \nabla^{2} \mathrm{c}
\end{gathered}
$$

Where, the velocity u, kinematic viscosity $v$, the density $\rho$, the pressure $\mathrm{p}$, concentration of water and water-dye $\mathrm{c}$, and diffusion coefficient of mixture $\mathrm{D}$.

For consistency of comparisons, pure water and a water-soluble dye are used for the mixing process. In this simulation laminar Newtonian flow with the condition of no-slip was taken and a species transport model is used. The dynamic viscosity of water $\mu=0.001 \mathrm{Ns} /$, density of water $\rho=1000 \mathrm{~kg} /$ and diffusion coefficient of mixture $\mathrm{D}=/ \mathrm{s}$ were obtained at standard temperature. The criteria of convergence for conservation of mass and momentum equations have opted as and for the species transport equation (conservation of energy) has opted as . For all simulations, the Pressure-Velocity Coupling under the SIMPLE scheme, the continuity and momentum equation under second-order upwind, and species transport equation under a third-order MUSCL Scheme were selected. At both inlets, the same velocity was employed and at the outlet, the value of pressure is taken zero gauge. Mass fractions of 0 (for pure water) at one inlet and 1 (for a solution of dye) at the second inlet were taken.

The concentration variance of the amalgam was estimated at any cross-section of the microchannel in the perpendicular direction of the flow. The concentration variance on any cross-section as characterized in Chen et al. (2016) is demonstrated as:

$$
\sigma=\sqrt{\frac{1}{N} \sum_{i=0}^{N}\left(\mathrm{C}_{\mathrm{i}}-\mathrm{C}_{\text {mean }}\right)^{2}}
$$

In the aloft mention equation, the number of point clouds on any cross-section $\mathrm{N}$, the concentration at $\mathrm{i}$ sampling point on any plane and the average value of concentrations The magnitude of the mixing index can figure out by the below mention equation:

$$
\mathrm{Mi}=1-\frac{\sigma}{\sigma_{\max }}
$$

In the aloft mention equation, the variance of concentration of amalgam at any plane $\sigma$ and the highest value of concentration variance. The range of mixing index value is zero to one where the values near to one indicate good mixing quality. 


\section{RESULTS OF CFD SIMULATION}

The evasions were toted in a simple $\mathrm{T}$ shape mixer, offset entrances $\mathrm{T}$ shape mixer and offset entrances $\mathrm{T}$ mixer with bend shape. Mixing index of all three geometry simple T shape mixer, offset entrances T shape mixer and offset entrances $\mathrm{T}$ micromixer with bend shape are compared and also screened the influence of Reynolds numbers.

\section{Grid Independence Test}

In this section, grid independency test for $\mathrm{T}$ junction micromixers has been performed. For this goal, four different mesh sizes with grid numbers 2556476, 3075127, 4523211, and 5019460 were simulated. Figure2 demonstrates mixing index calculation along mixing channel length for mesh elements 2556476, 3075127, 4523211, and 5019460.

There is a variation of $0.16 \%$ in the mixing index values at 4523211 and 5019460 elements respectively. The same tests were carried out for offset entrances $\mathrm{T}$ shape micromixer and offset entrances $\mathrm{T}$ micromixer with bend shape.

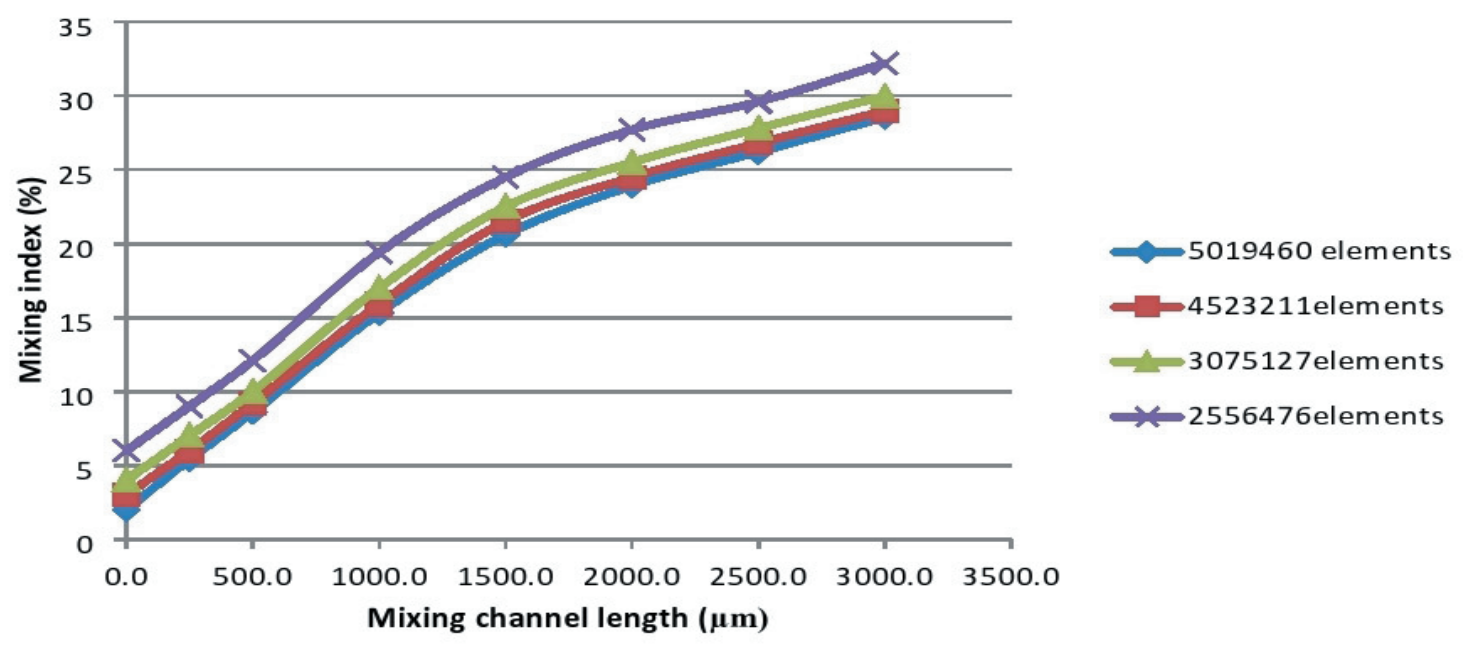

Figure 2. Mixing index percentage along mixing channel length for different mesh elements

\section{VALIDATION OF SIMULATION WORK}

This section consists of the validation of simulation work. The outcomes got by the current simulation were validated with the existing literature outcomes of Dundi et al.(2019). Figure3 shows a comparison of the current simulation and Dundi et al.(2019) work. The results show good agreement between the current simulation and Dundi et al.(2019) work. 


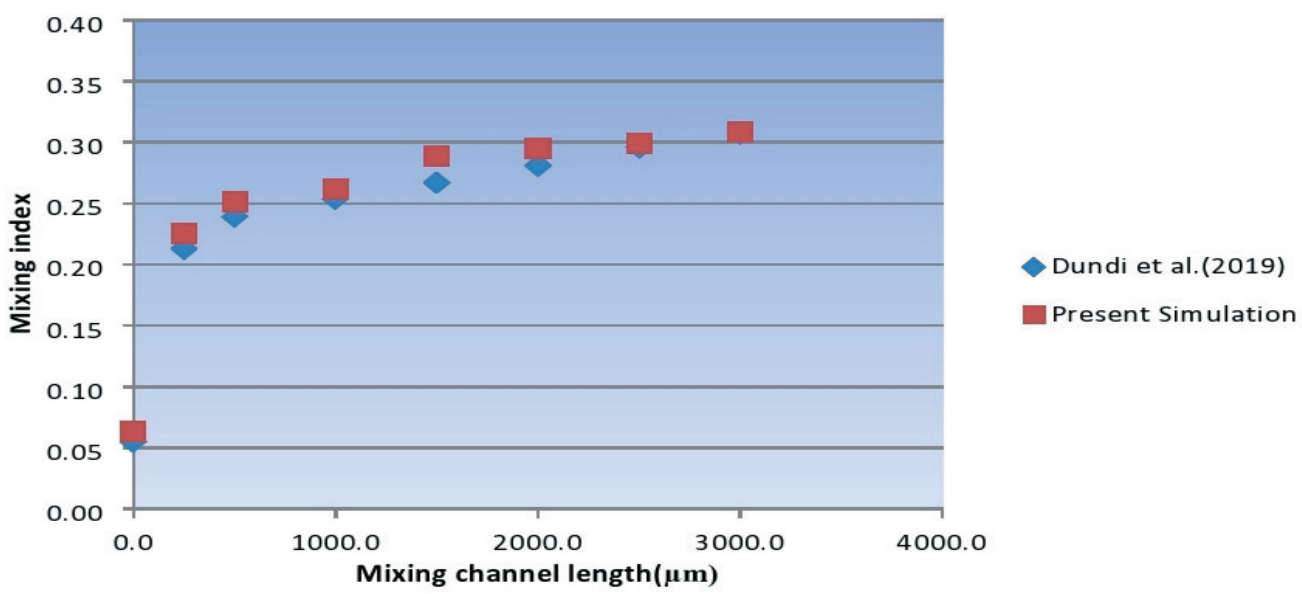

Figure 3. Comparison of mixing index of current simulation and consisting work

\section{INFLUENCE OF DESIGN}

Figure 4 demonstrates the concentration contours of simple $\mathrm{T}$ shape micromixer, offset inlets $\mathrm{T}$ shape micromixer and offset inlets $\mathrm{T}$ micromixer with bend shape, in which offset inlets $\mathrm{T}$ mixer with bend shape is found to be better than simple $\mathrm{T}$ shape mixer and offset inlets $\mathrm{T}$ shape mixer. The value of mixing index at the outlet for offset inlets $\mathrm{T}$ micromixer with bend shape is advanced $\left(M_{(i)}=0.514\right)$ than that of the offset inlets $\mathrm{T}$ shape mixer $\left(M_{i}=0.468\right)$ and simple T shape mixer $\left(M_{i}=0.280\right)$.

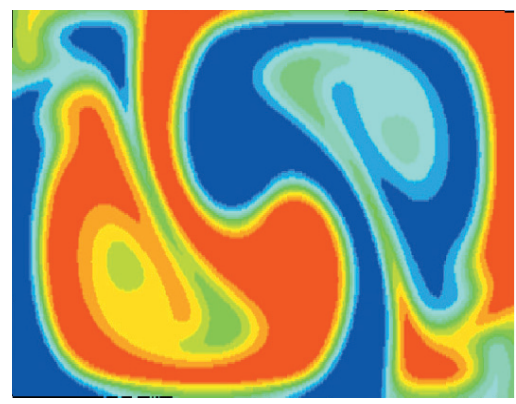

(a)

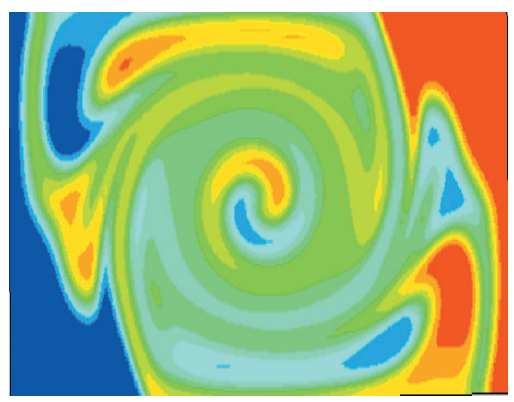

(b)

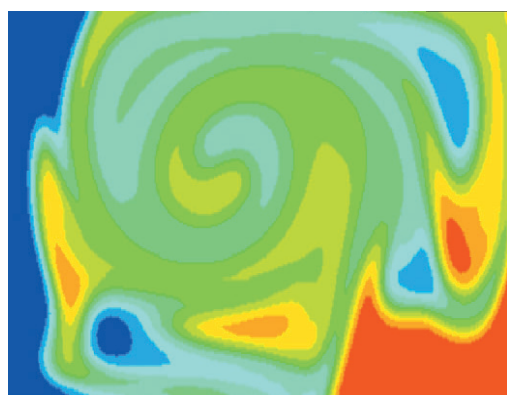

(c)

Figure 4. Concentration contours of $\mathrm{T}$ micromixer at outlet for (a) simple (b) offset inlets (c) offset inlets with bend structure at $\mathrm{Re}=500$. 


\section{INFLUENCE OF RAISING INLETS VELOCITY}

The influence of raising inlets velocity (Reynolds numbers) on mixing index in a simple $\mathrm{T}$ shape mixer, offset entrances T shape mixer and offset entrances T- mixer with bend shape was screened. For this intention, a simple T shape mixer, offset entrances $\mathrm{T}$ shape mixer and offset entrances $\mathrm{T}$ mixer with bend shape keeping the similar mixing channel length was simulated at varied Reynolds numbers extenting from 30 to 500 . Figure 5 demonstrates mixing quality numeration against Reynolds numbers for the simple $\mathrm{T}$ shape micromixer, offset entrances $\mathrm{T}$ shape micromixer and offset entrances T micromixer keeping bend shape. As demonstrated in figure 5, Mixing index in the offset entrances $\mathrm{T}$ micromixer with bend structure raises sharp with raising the Reynolds numbers.

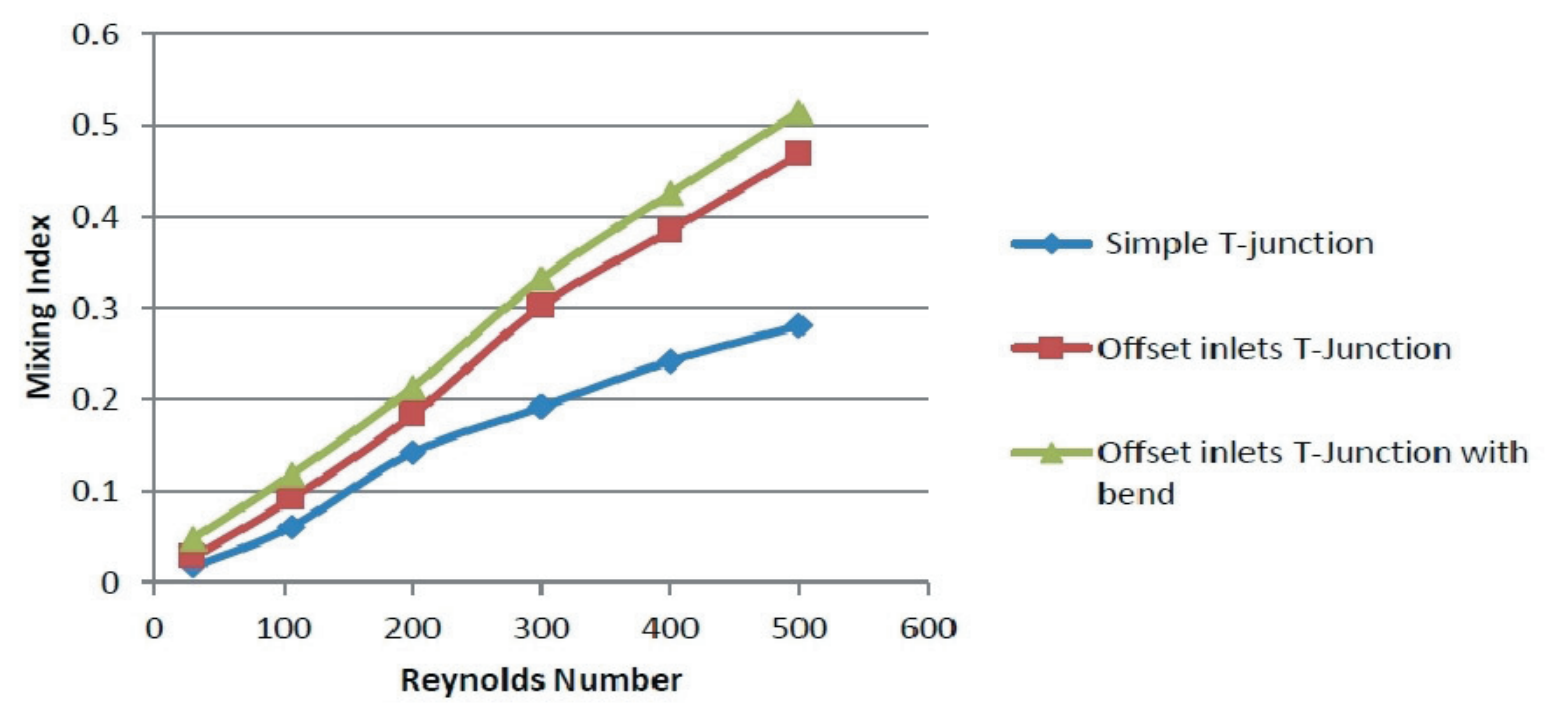

Figure5 Mixing index for the simple T shape micromixer, offset entrances $\mathrm{T}$ shape micromixer and offset entrances $\mathrm{T}$ mixer with bend shape at different Reynolds numbers.

From the above results, we see that the offset entrances T-mixer keeping bend shape is most efficient than the simple $\mathrm{T}$ shape mixer and offset inlets $\mathrm{T}$ shape mixer. In the case of passive micromixers, we need only the fluid pumping power, without any external powers; the calculated pressure drop indicates the consumed power in the system. Figure 6 demonstrates pressure fall in simple T-micromixer, offset entrances T-micromixer and offset entrances T-micromixer keeping bend shape at varied Reynolds numbers. As displayed in figure 6, pressure fall in the offset entrances T-micromixer keeping bend shape is narrowly advanced than the offset entrances T- micromixer and simple T-micromixer. Since chaotic advection is created in the micromixers by a bend or fold the mixing channel, this type of microchannels develops a secondary flow lead by centrifugal force, which motives an advanced pressure fall in the microchannel. Even, the mixing quality for simple $\mathrm{T}$ shape mixer, offset entrances $\mathrm{T}$ shape mixer and offset entrances T-micromixer keeping bend shape was evaluated along the mixing channel. Figure 7 demonstrates mixing index along mixing channel for simple $\mathrm{T}$ shape mixer, offset entrances $\mathrm{T}$ shape mixer and offset entrances T-micromixer keeping bend shape. As looked at this figure, the mixing index value increases with increasing down-channel length. 


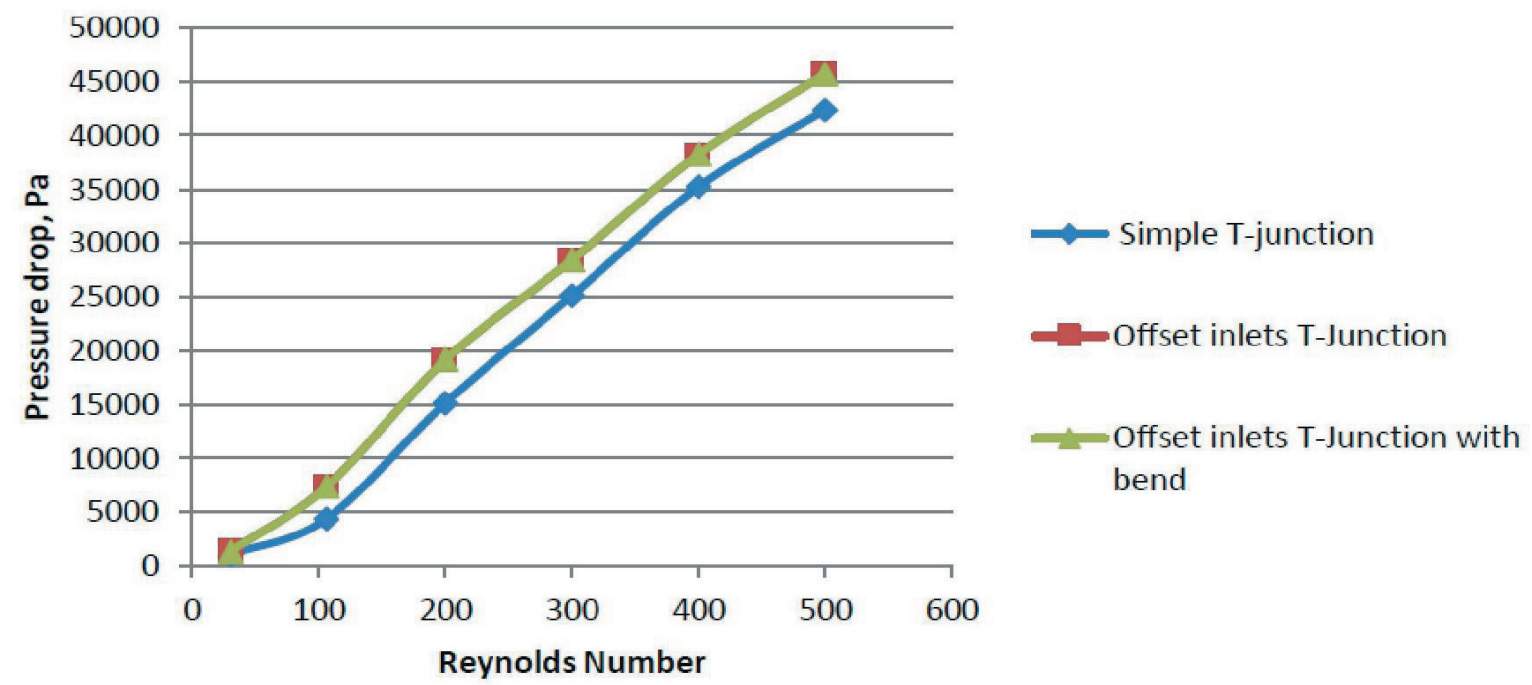

Figure 6. The pressure fall values in the simple $\mathrm{T}$ shape micromixer, offset inlets $\mathrm{T}$ shape micromixer and offset inlets T-mixer keeping bend shape at varied Re.

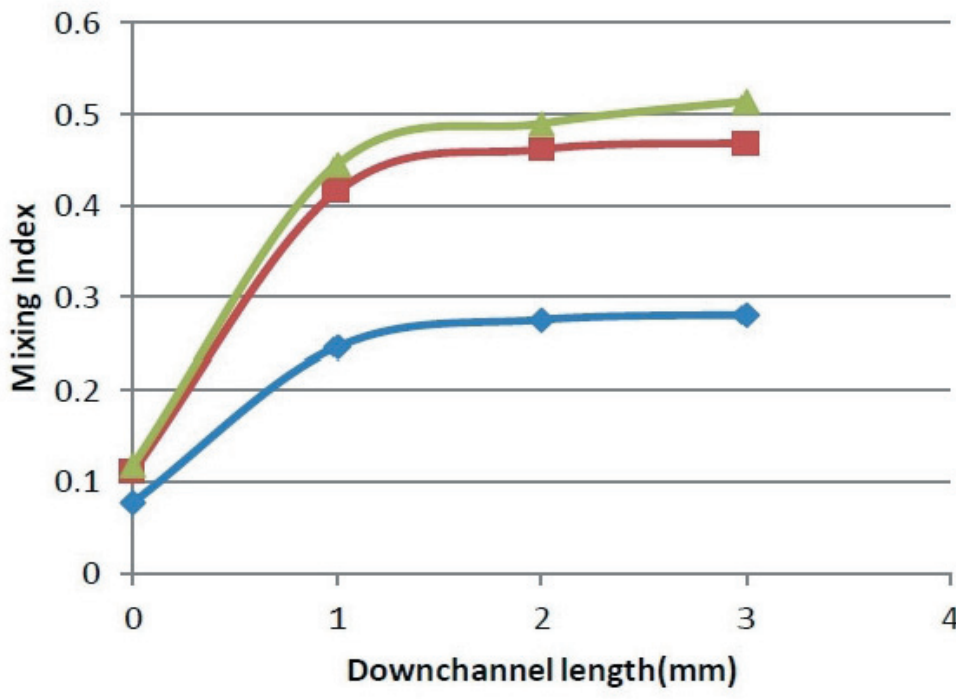

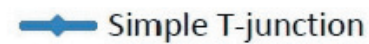

- Offset inlets T-Junction

$\longrightarrow$ Offset inlets T-Junction with bend

Figure 7. Mixing index versus the mixing channel length for simple T shape mixer, offset entrances $\mathrm{T}$ shape mixer and offset entrances T-mixer keeping bend shape. 


\section{CONCLUSIONS}

In this work, a simple T mixer, offset entrances $\mathrm{T}$ mixer and offset entrances $\mathrm{T}$ mixer keeping bend mixing channel have been simulated. It was obtained that offset entrances $\mathrm{T}$ mixer keeping bend structure yields an advanced mixing index than that of simple $\mathrm{T}$ mixer and offset entrances $\mathrm{T}$ mixer due to increased aspect ratio and chaotic advection. Pressure fall in offset entrances $\mathrm{T}$ mixer keeping bend structure is excess than simple $\mathrm{T}$ mixer but narrowly excess than offset entrances $\mathrm{T}$ mixer.

\section{ACKNOWLEDGMENT}

This work was supported by Delhi Technological University, Delhi.

\section{CONFLICT OF INTEREST}

The authors declare that there is no conflict of interest regarding the publication of this paper.

\section{REFERENCES}

Quiroz C. A., Azarbadegan A. \& Zangeneh M., 2016. Effect of channel aspect ratio of 3D T- mixer on flow patterns and convective mixing for a wide range of Reynolds Number. Sensors and Actuators B, 239 1153-76.

Quiroz C. A., Azarbadegan A. \& Zangeneh M., 2014. Evaluation of flow characteristics that give higher mixing performance in the 3-D T-mixer versus the typical T-mixer. Sensor and Actuators B, 202 1209-19.

Solehati N., Bae J. \& Sasmito A. P., 2014. Numerical investigation of mixing performance in microchannel T-junction with wavy structure. Computers and Fluids, 96 10-19.

Rahimi M., Akbari M., Parsamoghadam M. A. \& Alsairafi A. A., 2014.CFD study on effect of channel confluence angle on fluid flow pattern in asymmetrical shaped microchannels. Computers and Chemical Engg., 73 172-82.

Nguyen T. N. T., Kim M. C., Park J. S. \& Lee N. E., 2008. An effective passive microfluidic mixer utilizing chaotic advection. Sensors and Actuators B, 132 172-81.

Tsai T. H., Liou D. S., Kuo L. S. \& Chen P. H., 2009. Rapid mixing between Ferro- nanofluid and water in a semiactive Y-type micromixer. Sensors and Actuators A, 153 267-73.

Wong S. H., Ward M. C. L. \& Wharton C. W., 2004. Micro T-mixer as a rapid mixing micromixer. Sensors and Actuator B, 100 359-79.

Alam A. \& Kim K. Y., 2012. Mixing performance of a planar micromixer with circular chambers and crossing constriction channels. Sensors and Actuators B, 176 639-52.

Hossain S., Ansari M. A. \& Kim K. Y., 2009. Evaluation of the mixing performance of three passive micromixers. Chemical Engg. J., 150 492-501.

Chen X., Li T., Zeng H., Hu Z. \& Fu B., 2016. Numerical and experimental investigation on micromixers with serpentine microchannels. Int. J. of H. M. T, 98 131-40.

Hsieh S. S., Lin J. W. \& Chen J. H., 2013. Mixing efficiency of Y-type micromixers with different angles. Int. J. of Heat and Fluid Flows, 44 130-39.

Tokas S., Zunaid M. \& Ansari M. A., 2020. Numerical investigation of the performance of 3D-helical passive micromixer with Newtonian fluid and non-Newtonian fluid blood Asia-Pacific J. of Chemical Engg. 
Tokas S. \& Zunaid M., 2020. Computational analysis of passive mixing in T-micromixer with Non-newtonian blood. Int. J. of Mech. \& Prod. Engg. R and D, 9889-98.

Javed S. F. \& Zunaid M., 2020. Numerical investigation inside spiral passive micromixer using nanofluid. Int. J. of Mech. \& Prod. Engg. R and D, 9825-34.

Wang L., Wu W. \& Li X., 2013. Numerical and experimental investigation of mixing characteristics in the constructal tree-shaped microchannel. Int. J. of H. M. T, 67 1014-23.

Vatankhah P. \& Shamloo A., 2018. Parametric study on mixing process in an in-plane spiral micromixer utilizing chaotic advection. Analytica Chimica Acta, 1022 96-105.

Dauyeshova B. \& Monaco E., 2018. Numerical simulation of diffusion process T- shape micromixer using shanChen Lattice Boltzmann Method. Computers and Fluids, 167 229-40.

Veldurthi N., Chandel S., Bhave T. \& Bodas D., 2015.Computational fluid dynamic analysis of poly(dimethyl siloxane) magnetic actuator based micromixer. Sensors and Actuators B, 212 419-24.

Lobasov A.S. \& Minakov A.V., 2018.Analyzing mixing quality in a T-shaped micromixer for different fluids properties through numerical simulation.Chemical Engg. \& Processing, 124 11-23.

Liu X. \& Lu Y., 2019. Highly efficient and flexible preparation of water-dispersed Fe3O4 nanoclusters using a micromixer. Particuology, 45 42-48.

Chen X. \& Shen J., 2016. Numerical analysis of mixing behaviors of two types of E-shape micromixer. Int. J. of H. M. T, 106 593-600.

Haghighinia A. \& Movahedirad S., 2019. Fluid micro-mixing in a passive microchannel: Comparison of 2D and 3D numerical simulations. Int. J. of H. M. T, 139 907-16.

Rahmannezhad J. \& Mirbozorgi S. A., 2019. CFD analysis and RSM-based design optimization of novel grooved micromixers with obstructions. Int. J. of H. M. T, 140 483-97.

Chen X. \& Zhao Z., 2017. Numerical investigation on layout optimization of obstacles in a three- dimensional passive micromixer. Analytica Chimica Acta, 964 142-49.

Balasubramaniam L., Arayanarakool R., Marshall S. D., Li B., Lee P. S. \& Chen P. C. Y.,2017. Impact of cross-sectional geometry on mixing performance of spiral microfluidic channels characterized by swirling strength of Dean-vortices. J. of Micromechanics and Microengg.,27 095016.

Nazari M., Rashidi S. \& Esfahani J. A., 2019. Mixing process and mass transfer in a novel design of induced-charge, electrokinetic micromixer with a conductive mixing-chamber. Int.communication in H. M.T, 108104293.

Silva J. P., Santos A. D. \& Semiao V., 2017. Experimental characterization of pulsed Newtonian fluid flows inside T- shaped micromixers with variable inlets widths. Experimental Thermal and Fluid Science, 89 249-58.

Santana H. S., Tortola D. S., Jr. J. L. S. \& Taranto O. P., 2017. Biodiesel synthesis in micromixer with static elements. Energy Conversion and Management, 141 28-39.

Dundi T. M., Raju V. R. K. \& Chandramohan V.P., 2019. Numerical evaluation of swirl effect on liquid mixing in a passive T-micromixer.Australian Journal of Mechanical Engineering.

Boye T. G. E. \& Samuel O. D., 2020. Computer-based method of design and modeling of transient flow in crude oil pipeline system. Journal of Engg. Research, 8 219-39.

Sahnoune K., Benbrik A., Mansour A. S. \& Oussama R., 2020. Flow simulation and performance analysis of a drilling turbine. Journal of Engg. Research, 8 255-70.

Afzal A., A. D. M. S. \& R.K. A. R., 2019. Experimental investigation of thermal performance of engine coolant oil and water in helical coil heat exchanger. Journal of Engg. Research, 7 333-51. 